\title{
Research on Automatic Sprinkling and Feeding Machine for Nereis Farming
}

\author{
WU Ziyi * \\ Teaching Affairs Department \\ Suzhou Chien-Shiung Institute of Technology \\ Taicang, China \\ E-mail: 1015671421@qq.com \\ * Corresponding Author \\ CHEN Changlong \\ School of Mechanical Engineering \\ Yancheng Institute of Technology \\ Yancheng, China \\ E-mail: 1542062518@qq.com
}

\author{
HUI Weidong \\ School of Mechanical Engineering \\ Yancheng Institute of Industry Technology \\ Yancheng, China \\ E-mail: 120817713@qq.com
}

\author{
SHI Jingjing \\ School of Mechanical Engineering \\ Yancheng Institute of Technology \\ Yancheng, China \\ E-mail: 1052304455@qq.com
}

\begin{abstract}
An integrated machine with automatic sprinkling and feeding functions for Nereis farming was presented in this paper. Different from traditional manual feeding method, the machine is assembled on a vehicle chassis which is driven by engine and electricity. A cam mechanism is fixed on the back axle of the vehicle and achieve a synchronous motion with the vehicle. The cam mechanism pushes piston to move up-and-down to eject water into mixing tank. A four-bar mechanism was employed to shake food box, from which food fell into the same mixing tank. Key techniques and 3D models of the integrated machine were discribed in this paper.
\end{abstract}

Keywords-Nereis Farming; Integrated Machine; Automatic Sprinkling; Automatic Feeding; Cam Mechanism

\section{INTRODUCTION}

Nereis is a kind of segmented worms, which is generally inhabited in coastal mud and widely used as fishing bait. Nereis has become an emerging aquaculture object with a very optimistic outlook in industrialization due to its good adaptability, high nourishment value, high annual turnover rate and good economic returns, etc. However, due to its unique breeding habits, the special farming conditions and immature artificial farming technology, Nereis breeding scale of is not as great as it should be [1]. Nereis has a unique life, which just stretch out the front body a few centimeters outside its cave for food hunting. Therefore, the powder food must be uniformly casted into the whole pool. Currently, Nereis feeding relies entirely on manual feeding, which is very low in feeding efficiency and uneven in density of food casting. Manual feeding not only often leads to the deterioration of water environment, but also results in the overfeeding and food shortage of some Nereis.

With the continuous development of coast farming feeding technology, the requirements for the level of uniformity of feeding is getting higher, at the same time, the culture environment of high density also needs to reduce the pollution of the water. Therefore, in order to adapt to the development of Nereis farming, it is urgent to design and develop a kind of automatic sprinkling and feeding machine for Nereis farming, which is of high performance, efficiency and integration.

\section{RESEARCH STATUS OF RELATED TECHNOLOGIES}

China is both a major agricultural country and a forestry country, which needs a high application level of water-sprinkling and food-breeding technology. However, many such works are done manually. The disadvantages of manual method are obvious, which not only cause the waste of resources, but also lead to an unstable control degree, and the efficiency of water sprinkling is low. In view of the limitations of the above-mentioned system, $\mathrm{Li}$ D. proposed a kind of control system, which is called lawn intelligent sprinkler system based on ZigBee. The system is composed of the sensor nodes, the work station and the monitoring station. By testing, the system is of a reasonable structure and low cost, and works stably. For achieving the anticipated quota satisfactorily, it has a certain popularization value [2].

Liu J. et al. researched on the simulation of $0.2 \mathrm{MPa}$ water pressure under laboratory conditions, and had a detection of different nozzles in the $50 \mathrm{~cm}, 60 \mathrm{~cm}$ and $70 \mathrm{~cm}$ three kinds of spray intensity and the combination of the spray intensity and the combination of average spray intensity. The results show that the spray intensity of each spray nozzle decreases with the increase of the combined spacing, and the optimum combination of the combination is $1.7 \mathrm{~m}, 1.3 \sim 1.4 \mathrm{~m}, 1.6 \mathrm{~m}$ and $1.5 \sim 1.6 \mathrm{~m}[3]$.

At present, there are three domestic ways for crab cage aquaculture feeding: the first way is artificial feeding by punting, which depends on artificial experience. In this way the work efficiency is low, labor intensity is high, and there is no guarantee for the feeding uniformity. The second method is machine feeding, which only feed in the same location, and cannot guarantee the feeding 
uniformity. The third approach is vessel feeding machine, which needs workers cast bait into the pond [4]. This approach may easily pollute the water and is difficult to guarantee feeding uniformity, which has a strong randomness

In order to solve the feeding problem in river crab aquaculture, Sun Y., Zhao D., Hong J, et al. developed an automatic air propeller driven feeding system carried by a workboat and uniform feeding method. The crab feeding system is mainly composed of a workboat driven by air propellers, an automatic feeding device, an ARM main controller, a GPRS communication module and a GPS navigation device. This feeding device provides technology reference for further study of automatic uniform feeding on the whole pond in river crab aquaculture and other aquaculture needing for feeding (it has strong reference value for Nereis feeding too) along the pond or on the whole pond [5]. In this system, ARM main controller receives feeding task through the GPRS [6-7], automatic operates feeding path and direction.

\section{KEY TECHNIQUES FOR THE DESIGN OF THE NEREIS FEEDING MACHINE}

\section{A. Even Mixing Technique}

Even mixing is an important technique in Nereis feeding production, which has a significant impact on the animal production performance. If the bait's feeding is uneven, it easily leads to excessive or insufficient nutritional components in feeding. Therefore, the mixing evenness of feeding directly relates to the quality of the products, and also affects the long-term stable and healthy development of animal husbandry [8].

Serum mixed with powder feed and water can easily separate and settle in a static state. Therefore, in order to keep the feed liquid uniformity in the process of delivery, the mixed slurry system is remodeled. The feed is stored in a feed box with a fixed frequency vibration, in which water can be released into the tank through the hole. Water tank and steel agitator component compose the slurry mixing system. The cone bucket shaped steel drum is designed as the bottom of the tank by 15 degrees, which can effectively avoid the feed liquid rotating stall.

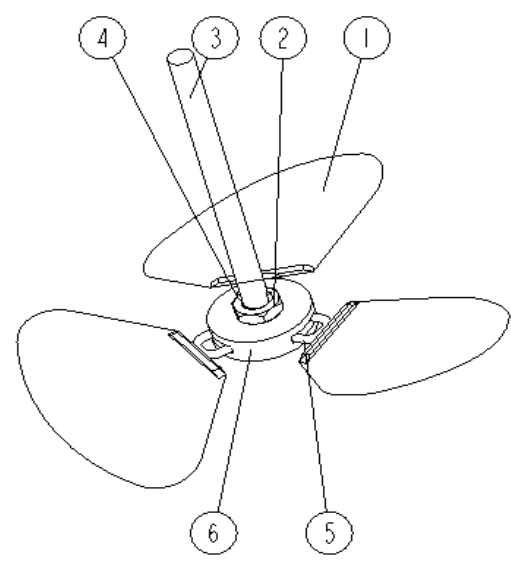

Figure1. Structure diagram of agitator

The mixer is composed of a motor, a rotating shaft and an impeller, as shown in Figure 1. The rotating shaft and the impeller are maintained at a certain speed, and the impact of the water flow on the mixture is used to ensure the uniformity of the liquid concentration in the mixing chamber. The agitator is composed of blade (1), lock nut (2), gravity shaft (3), stop pin (4), snap ring (5), mixing head (6).

\section{B. Nozzle Deposition Technique Based on Multi Cycle Body}

Pipe is a common tool in daily life, the connection between two pipes need a pipe connecting part. Valves and pipes are mainly used for conveying a variety of physical state of the device. The pipe is always made of hard materials and it is collapsible, or others are made of leather and they are foldable, there is a disadvantage that the pipe is unable to realize floating automatic operation. Therefore, it is urgent to develop a kind of method which can realize the automatic operation, and can realize the folding of the sprinkling pipe.

This technique is mainly composed of multi cycle body and annular nozzle:

a) The diameter of cycle body is slightly smaller than the diameter of the nozzle in this technique, ball rolls forward under the dual affects of siphon and water; it forms a torrent between the water and nozzle in this process, stirring up sediments and washes away sediments. The matrix of multi cycle body is classified as fan type and hollow ball, an "S" type rubber strip is installed on its surface, it can play a role of dredging and protection nozzle. Under the combined effects on the body of the loop flow momentum and uneven flow, elimination effect of nozzle sediments and the smoothness of cycle body are improved.

b) The feeding nozzle is designed into the annular nozzle. Generally speaking, the circular throat type annular nozzle array is more practical value compared with the smooth characteristic angle [9]. The annular ball can reduce the delivery quantity of steel ball, the reverse flow near the closed end regions will lead to water static or slow down, it can reduce the water flow at closed end of the nozzle by the use of annular nozzle, and avoid feeding deposition near the closed end the nozzle. As shown in Figure 2, this is an annular nozzle.

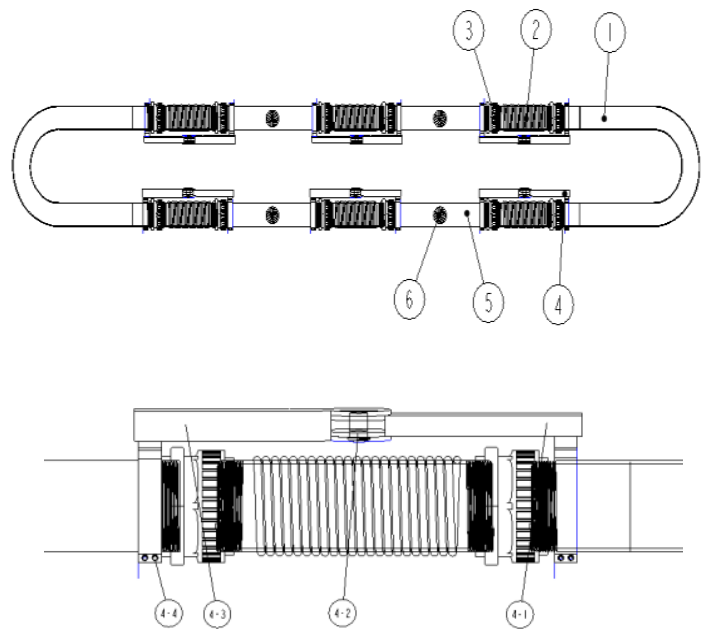

Figure 2. Structure diagram of annular nozzle 
As shown in Figure 2, the annular nozzle is composed of $U$ type pipe (1), short tube (2), connecting nut (3), supporting seat (4), long tube (5) and porous nozzle (6). When the U type pipe I working, the supporting rod is 180 degrees, pipe is horizontal, and the pipe will not bent by the interference fit of support rod and connecting nut; when the $U$ pipe does not work, the connection rods is bent, $U$ type pipe will fold at the same time.

\section{DESIGN OF THE INTEGRATED MACHINE}

The design of the whole machine includes the specific design of the first two techniques and the overall environmental adaptability design.

a) Specific design: The design of hydraulic system is mainly including the single pump, to switch the water and transport slurry state by the use of multi control valve. The length of the annular nozzle depends on the width of the pool, refer to both ends of the nozzle and a half circle diameter, the diameter of the annular nozzle is generally $10 \mathrm{~cm}$. In order to prevent the fan's oblique; the boss is increased in the fan type cycle body, the car walk and hydraulic valve control are controlled by electronic control system.

b) Adaptive design: The width of Electric two wheel vehicle shall be not more than Nereis feeding pool distance, the annular nozzle is settled on the both sides of vehicle outward extension by $3 \sim 5 \mathrm{~m}$, in order to maintain the balance of integrated machine, a pulley is installed at the top of the nozzle. The control valve is used to control the single and bidirectional opening of the nozzle. The nozzle is designed for two cycles, which can work independently, and can be folded and reduce the space occupancy rate of integrated machine.

Since the design concept is integrated with analysis, the design parameters can include not only the geometry parameters, but also quantities such as material properties, loads, and boundary conditions [10]. In order to facilitate the subsequent model modification, design scheme improvement and model checking, the design of the component parts is carried out by using parametric modeling technology. The structural arrangement of the integrated machine is shown in Figure 3.

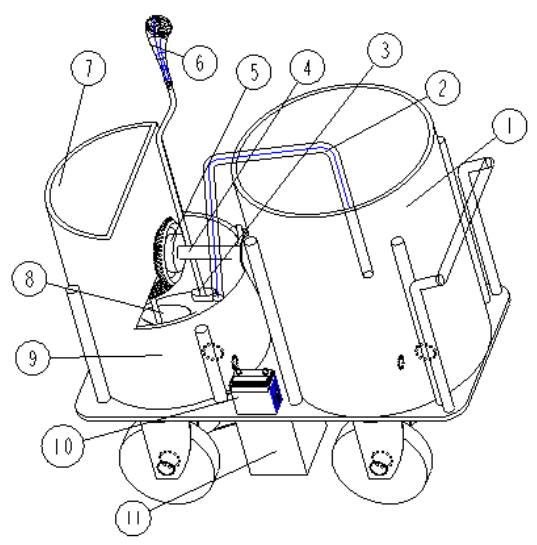

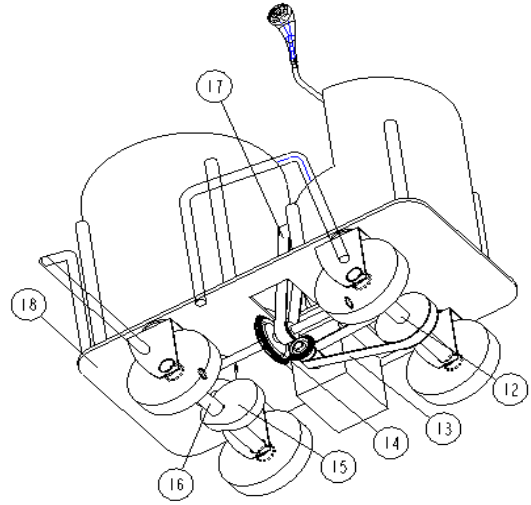

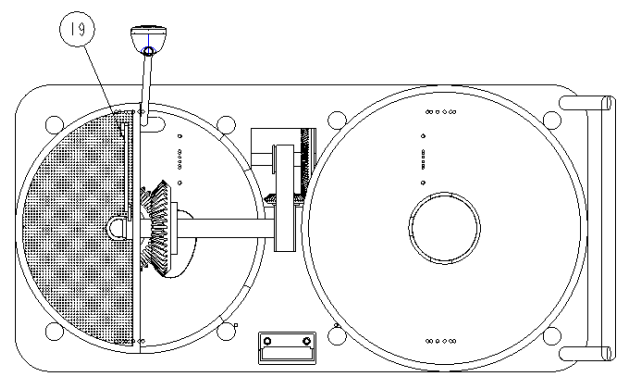

Figure 3. Layout structure of integrated machine

In Figure 3, the different number label indicates different parts or components. 1 delegates big water tank, 2 water pipe, 3 pump, 4 gear shaft, 5 gear, 6 sprayer, 7 feed box, 8 agitator arm, 9 agitator tank, 10 battery, 11 engine, 12 front axle, 13 belt wheel, 14 bevel gear, 15 cam, 16 back axle, 17 belt wheel, 18 body of the vehicle, and 19 crank and rocker mechanism. The 3D model of the machine is shown as Figure 4.

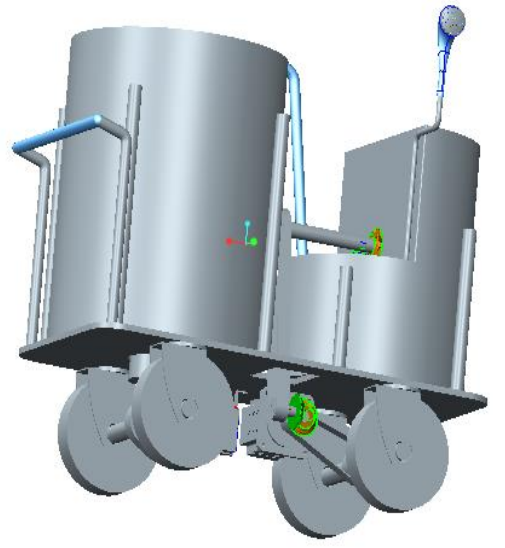

Figure 4. 3D model of the integrated machine

\section{PARAMETER DESIGN}

\section{A. Main Design conditions}

Assuming the Nereis farming pond is rectangle one with an area of $60 \times 50 \mathrm{~m}^{2}$, and there is a $1.5 \mathrm{~m}$ wide road around the pond. The integrated machine is driven by electricity from battery. The driving direction is controlled manually. The voltage of the electricity is $220 \mathrm{v}$. The diameter and height of the cylindrical water tank is $0.8 \mathrm{~m}$ and $1 \mathrm{~m}$ respectively. 


\section{B. Engine Choice}

The total mass of the water tank, which is fully loaded, is calculated by the following equation:

$$
m=\rho V+m_{f}
$$

Where, $m$ is the total mass of water tank, $\rho$ the density of water, $V$ the volume of the water tank, $m_{f}$ the total mass of food that will be added into the water tank.

The theoretical power of the engine on the machine is calculated by the following equation:

$$
P=G v
$$

Where, $G$ is the total weight of the vehicle that is fully loaded with water and food, and $v$ is transmission speed of the $\mathrm{V}$ belt.

The transmission efficiencies of $\mathrm{V}$ belt and bevel gear pair are about 0.96 and 0.97 respectively. Therefore, the minimum rated power of the chosen engine can be acquired by the following equation:

$$
P_{e}=\frac{P}{\eta_{1} \eta_{2}}
$$

Where $\eta_{1}$ and $\eta_{2}$ are the transmission efficiency of $\mathrm{V}$ belt and bevel gear pair respectively.

\section{Eelt Transmission}

Belt transmission relies on the friction between the belt and belt wheel to transfer movement and power. We choose common $\mathrm{V}$ belt as the transmission part, because it can alleviate the rigid impact of load and only produces particularly little noise, and attains high precision. When the transmission system is overloaded, the belt will skid to avoid the destruction of the system.

For belt conveyor load changes little, the driving power of the belt transmission system can be calculated by the following equation:

$$
P_{c}=K_{A} \cdot P
$$

Where, $K_{A}$ is performance coefficient, which can be found from the design handbook.

The moving speed of the belt can be calculated by the following equation:

$$
v=\frac{\pi d_{1} n_{1}}{60 \times 1000}
$$

Where, $d_{1}$ is the diameter of driving belt wheel, and $n_{1}$ the rotation speed of the engine. And, the diameter of the driven belt wheel can be attained by the following equation.

$$
d_{2}=i_{1} d_{1}(1-\varepsilon)
$$

\section{Bevel Gear Transmission Design}

Bevel gear mechanism is often used to transfer movement and power between intersecting axles. Usually the parameters of big end of bevel gear are used as the calculation and measurement reference values. In this design, the bevel gears are made of $45 \#$ steel, through tempering and heat treatment, and the hardness of tooth surface close to $255 \mathrm{HBS}$. According to the hardness of tooth surface, we can get $\sigma_{H \mathrm{lim} 1}=600 \mathrm{MPa}$, $\sigma_{H \lim 2}=560 M P a$ and $S_{H}=1$.

$$
\left\{\begin{array}{c}
{\left[\sigma_{H}\right]=\frac{\sigma_{H \lim } \cdot K_{N}}{S_{H}}} \\
{\left[\sigma_{F 1}\right]=\frac{\sigma_{F \lim b} \cdot K_{F c} \cdot K_{F L}}{S_{F}}} \\
{\left[\sigma_{F 2}\right]=\frac{\sigma_{F \lim b} \cdot K_{F c} \cdot K_{F L}}{S_{F}}}
\end{array}\right.
$$

The transmission torque can be calculated by the following equation.

$$
T_{1}=\frac{9549 \times 10^{3} P_{1}}{n_{1}}
$$

Thus the diameter of the driven bevel gear is expressed by the following equation.

$$
d_{m 1}=K_{d} \sqrt[3]{\frac{T_{1} K_{\beta}}{0.85 \varphi_{d}\left[\sigma_{H}\right]^{2}} \cdot \sqrt{\frac{u^{2}+1}{u}}}
$$

In a similar way, the parameters of driving bevel gear can be calculated through (7) (9). In (9), the value of $K_{d}$ can be attained by the following equation.

$$
K_{d}=\sqrt[3]{2\left(Z_{H} Z_{E} Z_{\varepsilon}\right)^{2} K_{v}}
$$

The module of the bevel gear is also an important parameter, which can be calculated by the following equation:

$$
m_{1}=\sqrt[3]{\frac{2 T_{1} K_{\beta} K_{v} Y_{F}}{0.85 Z_{1} \varphi_{m}\left[\sigma_{F}\right]}} \cdot R(R-0.5 b)
$$

The tooth number of the bevel gear is easily acquired by the following equation:

$$
Z_{1}=\frac{d_{1}}{m_{1}}
$$

\section{E. Cam and Piston}

According to the movement of followers, cam mechanisms are mainly divided into two groups: moving followers and oscillating followers. The movement of oscillating rollers is superfluous, because it only cause a local freedom. However, the rotation of rollers can switch the sliding friction between cam and roller into rolling friction, which greatly reduces the wear of cam mechanism 
and transfer larger power. The cam contour curve is designed through reverse rotation method. Reverse rotation method principle is that a reverse device is assembled on the cam, which do not change the relative motion between the components. Through this mechanism, the cam mechanism has an angular velocity which is equal to the angular velocity of the cam, but different in rotation direction. In this case, the cam keeps stationary and the follower can rotate around the rotation center of the rack in a reverse rotation velocity. The contour curve of the cam is designed by the following steps:

- Step 1: Select rational scale $\mu$, draw out the displacement line of the follower, and divide the horizontal coordinate of the displacement curve into several equal parts. The dividing points are marked with $1,2,3, \cdots$.

- Step 2: Select the same scale $\mu 1$ and choose the center of the circle and choose right radius, draw the cam's basic circle. Calculate and draw the follower's initial position through follower's offset direction and eccentricity e. The intersection point of position line and base circle is coincided with initial position on follower's tip.

- Step 3: Choose the follower's initial position line and the center of the circle. The tangent point of the offset circle is $\mathrm{K}$, which circle radius is offset distance.

- Step 4: Chose point K as the initial point and use the sub-section point of displacement graph as reference. Divide the offset circle into equivalence shares in reverse direction; get sub-section points 1 , $2,3 \cdots$ Draw rays through each sub-section points that tangent with offset circle. The intersection points of tangent rays and base circle are marked as $\mathrm{C} 1, \mathrm{C} 2, \mathrm{C} 3 \cdots \mathrm{C} 11$.

- Step 5: On the completed tangent rays, intercept line segments that separately equals to displacement coordinates from the outside of the base circle. The positions of the follower's tip are these points' position in the counter-rotating process.

- Step 6: The cam contour curve that needs to be designed is smooth connect points marked as B0, $\mathrm{B} 1, \mathrm{~B} 2 \cdots, \mathrm{B} 11$ in serial and format the cam mechanism of integrated machine.

\section{CONCLUSIONS}

In order to solve remove the problem of the blade and the stirring head and make up for the supporting and folding at the same time of traditional pipeline, this research develops an automatic sprinkling water and feeding integrated machine, the device can mix powder feed and water, and sprinkles them uniformly into the pool, the machine can sprinkle water and feed uniformly at the same time, greatly enhances the Nereis feeding efficiency, and improves the mechanization, intelligent, informationbased of the coastal aquaculture.

\section{ACKNOWLEDGMENT}

The authors gratefully acknowledge the support by the 2014 Taicang Research Foundation for Scientific and Technological Project on the Research and Development of the intelligent control system of Nereis Automatic water-spraying and bait-casting machine.

\section{REFERENCES}

[1] D. Li, "Effects of different environmental conditions on the larval development of perinereis aibuhitensis," Shandong: Ocean University of China, 2014.

[2] Z. Liu, "One kind system which is called lawn intelligent sprinkler based on ZigBee," Jiangsu, Jiangsu University of Science and Technology, 2013.

[3] J. Zhang, J. Wu, and T. Mao et al, "Optimization of spray combination scheme of open type milk cow house spray cooling system," Chinese cow, vol.22, issue 6, pp. 34-37, 2014.

[4] R. Tang, H. Zou and T. Tang et al, "Design of an automatic feeding boat and its control system," Fishery Modernization, vol.40, no.6, pp.30-35, 2013.

[5] Y. Sun, D. Zhao, and J. Hong, et al, "Design of automatic and uniform feeding system carried by workboat and effect test for raising river crab," Journal of agricultural engineering, vol.31, no.6, pp. 31-38, 2015.

[6] R. J. Bates (Bud), "Technology and Application for GPRS," Beijing: People's Post Press, 2004.

[7] D. Tamara, "Remote Communication," Beijing: Tsinghua University Press, 2004.

[8] C. Han, "The Determination About Uniformity Mixing And Moisture Content of FeedBased on Near Infrared Spectroscopic Technology," Inner Mongolia: Inner Mongolia Agricultural University, 2014.

[9] D. Jin, W. Liu, X. Chen et al, "Comparative analysis of annular nozzle and linear nozzle in CW DF/HF chemical laser," Laser \& Infrared, vol.40, no.11, pp.1186-1190, 2010.

[10] M. Hsua, C. Wanga, A. J. Herremaa, et al, "An interactive geometry modeling and parametric design platform for isogeometric analysis," Computers \& Mathematics with Applications, vol.002, no.4, 2015. 